\title{
Evaluation of the Divergence of a Thermal Neutron Beam using a Position Sensitive Detector
}

\author{
Gevaldo L. de Almeida, Maria Ines Silvani, Rosanne C. A. A. Furieri, \\ Instituto de Engenharia Nuclear - CNEN, C.P. 68550, \\ Ilha do Fundão, 21945-970, Rio de Janeiro, RJ, Brazil \\ Marcelo J. Gonçalves, and Ricardo Tadeu Lopes \\ Universidade Federal do Rio de Janeiro - COPPE, Bloco 6, 21945-970, Rio de Janeiro, RJ, Brazil
}

Received on 6 July, 2005

\begin{abstract}
The divergence of the thermal neutron beam available at the main outlet channel of the Argonauta Reactor in the Instituto de Engenharia Nuclear has been measured by using a Position Sensitive Detector. The measurement process addressing a proper beam characterization has been conducted under several geometric arrangements, including attempts to reduce the beam divergence by using a honeycomb-type gadolinium collimator. Since the detector presents finite resolution, each measured position spectrum is a convolution of the true Rocking Curve expressing the beam divergence with the function that describes the detector response. Therefore, those spectra have been submitted to an unfolding process to recover the concealed Rocking Curve. Prior to undertake this task however, the detector Line Spread Function - LSF was determined as required by the unfolding process. This was done by taking the response of the detector to a line source simulated by a narrow slit-collimator placed at several distances from the detector window. After a Lorentzian fitting to the acquired position spectra, the related FWHM were plotted against the source-detector distance. An extrapolation to zero furnishes then the width of the unknown LSF expressed as a normalized Lorentz function, which was then incorporated to a computer program developed to perform the unfolding. The final Rocking Curve emerges then as a normalized Lorentzian function properly defined by its width and a zero offset, showing that the beam diverges about 2.2 $\mathrm{cm}$ in 1 meter. The honeycomb collimator could not reduce this divergence due to its low aspect ratio.
\end{abstract}

\section{INTRODUCTION}

A full characterization of a neutron field requires the knowledge of several parameters such as its energy spectrum furnishing the contribution of thermal and epithermal neutrons, the neutron-to-gamma ratio, the beam intensity and its divergence expressed as its related Rocking Curve. All these parameters depend upon the specific arrangement used to moderate and collimate the neutron beam. The knowledge of the beam divergence is specially important whenever the direction of the incoming neutron plays a significant role in an experiment or measurement process, as in the Computer Assisted Tomography - CAT for instance, a growing field of non-destructive analysis in science and engineering where the image-reconstructing algorithms need this information to perform their tasks properly. Recent works [1-5] concerning the development and application of a Position Sensitive Detector - PSD to thermal neutron tomographic systems stimulated a work [6] which has shown the need to determine the divergence of the neutron beam and the viability to accomplish this task by using that kind of detector.

\section{FUNDAMENTALS}

While a non-divergent beam would cast a point-like shadow on a 2D-detector, a divergent one would draw a blurred spot with a higher density occurring at its center. An ideal detector would response to this neutron distribution with fidelity reproducing the very same pattern. A cross-section of the spatial distribution yields the Rocking Curve, henceforth in this work called RC for short.

For the particular case when the RC is independent from the spatial orientation, or when it is only needed for a specific one, then an 1D-PSD with its axis aimed at the desired direction and a slit collimator simulating a line source placed at a given distance from the detector and perpendicular to its axis could be used to assess the RC.

An infinitely narrow slit-collimator would be required to simulate a line source, but since this is unfeasible, in practice a certain finite aperture should be allowed. Under such a constraint, the single true RCs produced by the infinity number of imaginary line sources constituting the slit would convolute with each other to yield a curve broader than the true RC. Utilizing however a large detector-to-collimator distance this undesirable effect can be minimized to an acceptable level.

Besides this interference, the detector itself will spoil that RC through the convolution with its own Line Spread Function-LSF broadening the RC even more. This is a major problem specially for a low divergence beam, as it would preclude a direct determination of the RC.

This difficulty could be mitigated by placing the detector very far away from the collimator but practical limits would soon emerge as low counting statistics and detector length limitations. Indeed the detector should be long enough to contain a substantial portion of the RC, which would grow with the distance.

Therefore, the approach used in this work consist in the experimental determination of the detector LSF and the convoluted $\mathrm{RC}$, followed by a mathematical unfolding to recover the concealed true RC. 


\section{EXPERIMENTAL}

The experimental arrangement used to carry out the measurements to determine the LSF and the convoluted RC is shown in Fig.1. Further details concerning the PSD, its basic structure, operational principles and the associated ancillary electronics can be found elsewhere [1-4].

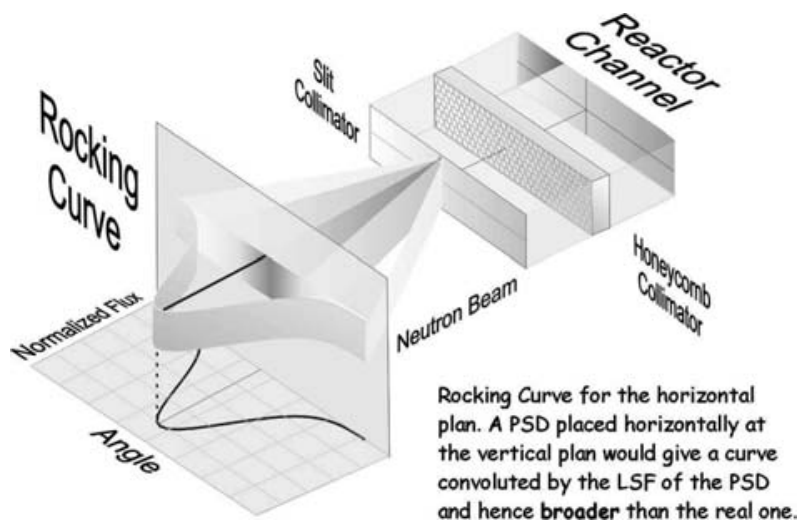

FIG. 1: Experimental arrangement used to determine the LSF and the RC. The beam divergence behavior for a given spatial direction is expressed by its Rocking Curve.

The neutrons emerging from the core of the Argonauta Reactor at the Instituto de Engenharia Nuclear are collimated with graphite blocks inside that channel. A $25 \mathrm{~mm}$-deep honeycomb-type gadolinium collimator has been used as an attempt to improve the beam parallelism. Since the side of the hexagon cell is $2 \mathrm{~mm}$-long, the collimator aspect ratio corresponds to an angle within the range 8-9 degrees.

The cadmium slit collimator simulates a line source emitting thermal neutrons. The beam divergence is exaggerated for illustration purposes only. Actually, it diverges only about $2.2 \mathrm{~cm}$ in 1 meter. The honeycomb collimator didn't change significantly this figure, for its aspect ratio was not tight enough for this divergence magnitude.

\section{Determination of the Line Spread Function}

The response of a PSD to a parallel infinitely narrow beam hitting it at a plane perpendicular to its axis is a somewhat blurred image instead of a sharp line. A plot of the apparent beam intensity hitting the detector along its axis constitutes the Line Spread Function-LSF [7].

There is thus a major difficulty to obtain the LSF: the production a parallel beam which additionally should be infinitely narrow. The first requirement can be reasonable fulfilled by using a high aspect ratio collimator or through the extrapolation procedure used in this work as follows.

In order to eliminate the impact of the unknown beam divergence, the detector-collimator gap should be zeroed. This zero-gap however cannot be reached due to the physical dimension of the detector itself. Indeed, the events registered by the detector occurs somewhere within the volume of its ionization chamber. Besides that, the thickness of the de- tector window adds an extra distance between the site where the events occurs and the slit collimator. Therefore, there is always a non-zero unknown gap allowing the divergence to play its unavoidable and undesirable role.

Assuming that the average event takes place at the middle of the ionization chamber, i.e., in the neighborhood of the anode wire it is reasonable to extrapolate the straight line expressing the apparent FWHM of the LSF versus distance to the anode to get the FWHM there. This value would not be affected by the beam divergence except that associated to the distance between the detector front face - where the collimator is placed - and the anode wire.

To carry out the above mentioned extrapolation, several spectra have been taken under different detector-to-collimator distances as shown in Fig.2.

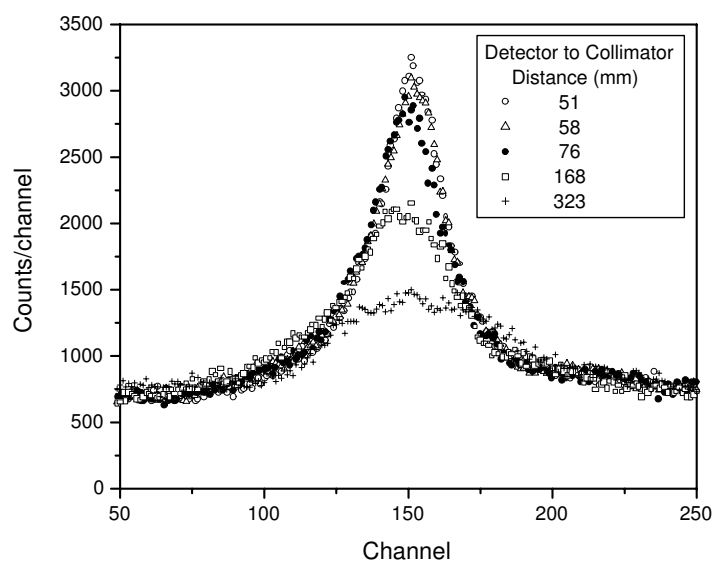

FIG. 2: Position spectra for several Detector-Collimator distances. The $51 \mathrm{~mm}$ distance corresponds to the collimator placed directly on the detector window, assuming that the effective detector position corresponds to that of its anode wire.

After a Lorentzian fitting, the width $\mathrm{w}$ for those spectra were plotted against that distance as shown in Fig.3. The intercept of a straight line fit with the vertical line at $\mathrm{x}=0$ - where the anode is located - as zoomed in the Fig. 4, furnishes the width of the Lorentzian expressing the detector LSF. The performed extrapolation solely corrects the influence of the beam divergence. The obtained Lorentzian still exhibits a FWHM, $w_{s}$, which is larger than the value $w_{0}$ produced by an infinitely narrow beam. Since the width $w_{c}$ of the slit is known, one can recover $w_{0}$, as follows:

$$
w_{0}=\left|\sqrt{\left(w_{s}^{2}-w_{c}^{2}\right)}\right|
$$

and the normalized Lorentzian with a zero offset is written as:

$$
y=\frac{y_{0}}{4\left(x-x_{0}\right)^{2}+w_{0}^{2}}
$$

where $x_{0}$ is the central channel determined by the non-linear fitting. 
It is worthwhile to stress that the real LSF profile remains unknown, for rigorously no experimental data is available for $\mathrm{x}=0$. Nevertheless, a Lorentzian function is a fair good approximation. Gaussians functions have been as well tested, but the chi-square and correlation were worst.

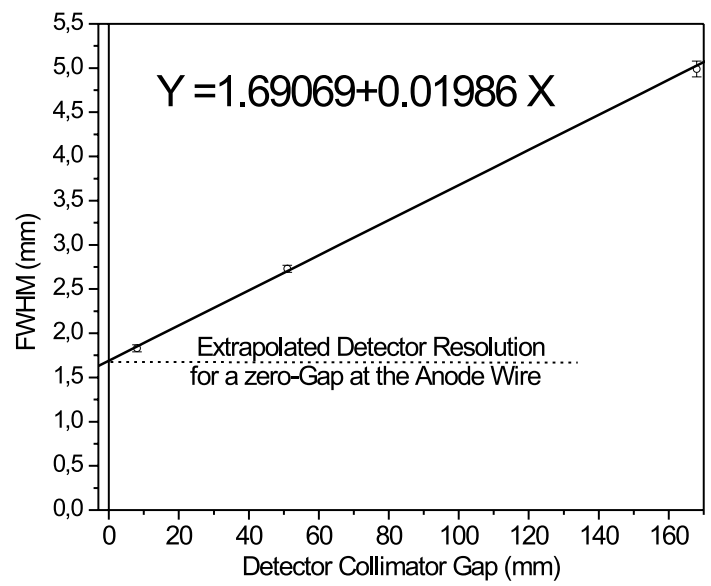

FIG. 3: The FWHM growth with the collimator-detector gap indicates a divergent beam. The zero-gap furnishes a LSF width of 1.69 $\mathrm{mm}$ after the straight line fit.

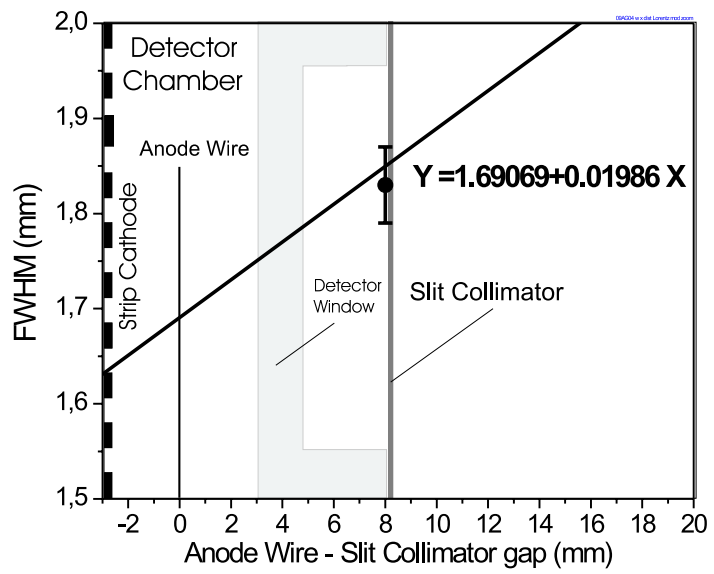

FIG. 4: Zoom of Fig.3 in the detector neighborhood, exhibiting its cross-section. The LSF width is determined assuming that the average ionization occurs at the anode.

\section{Determination of the Convoluted Rocking Curve}

All the spectra shown in Fig. 2 are convoluted RCs. The growth of the spectra width with the detector-collimator gap is a pure geometric effect caused by the beam divergence. When converted to the same gap they exhibit the same width as shown in Fig. 5, where they were normalized to make the comparison easier.

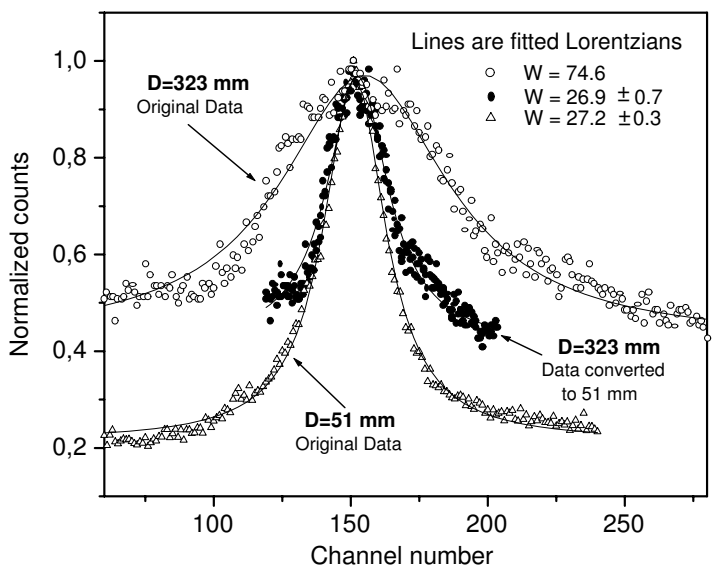

FIG. 5: Normalized position spectra for two different detectorcollimator gaps confirms a beam divergence. The width of both spectra coincide when converted to the same gap, showing the pure geometric character of the observed broadening.

The larger offset yielded by the larger gap is caused by the normalization process. The unfolding in the next section will be performed by using both the LSF and the convoluted RC with a zero-offset.

\section{MATHEMATICAL UNFOLDING}

A single point of the Rocking Curve at a given position can be regarded as a very sharp peak reminding a Dirac function having however a finite amplitude. Due to their finite resolution, no detector can reproduce such a sudden event, which is degraded into a bell-shaped curve resembling a Gaussian or Lorentzian with an area equivalent to that original amplitude. All the other points of the original spectrum have as well their own spoiled curves with their tails invading all the spectrum and contributing to the final amplitude of every single point. This convolution is mathematically expressed as:

$$
\vec{C}=\mathbf{A} \cdot \vec{Y}
$$

where:

$\vec{C}=$ Convoluted $\mathrm{RC}$ vector (experimentally acquired). $\mathbf{A}=$ Matrix of the coefficients for the linear system.

$\vec{Y}=$ Unfolded RC vector (unknown).

The matrix elements for a convolution involving two normalized Lorentzian functions, both having a zero offset, are readily deduced as:

$a_{i}^{j}=\frac{2 \cdot w}{1+\operatorname{Int}\left(2^{-j}\right)} \cdot\left\{\left[4(i-j)^{2}+w^{2}\right]^{-1}+\left[4(i+j)^{2}+w^{2}\right]^{-1}\right\}$ 
where:

$i, j=0,1,2,3, \ldots \mathrm{m}$.

$m+1=$ Number of channels in the spectrum

$w=$ Detector resolution in channels (LSF width).

Int $=$ Integer part.

It is easily figured out that the better the detector resolution, the closer the convoluted and unfolded curves would stay from each other.

\section{RESULTS AND CONCLUSIONS}

The unfolded RCs determined with and without the honeycomb collimator are shown in Fig. 6, where the original convoluted RCs (Lorentzian fittings to the experimental data) are as well presented for comparison. After the unfolding, the horizontal scale originally expressed in channels, was transformed into angles by using the spectrum gain (channel/mm) and the detector-collimator gap used to acquire the position spectrum. One can observe that the impact of the collimator on the RC is very slight. This happens because its aspect ratio corresponds to an angle in the range $8-9^{\circ}$, while the $\mathrm{RC}$ has a FWHM of $1^{\circ} 16$, i.e., the beam diverges roughly $2.2 \mathrm{~cm}$ in 1 $\mathrm{m}$. These figures refer to a divergence in the horizontal plan.
For other plans a somewhat different profile cannot be ruled out since the reactor channel has a rectangular cross-section.

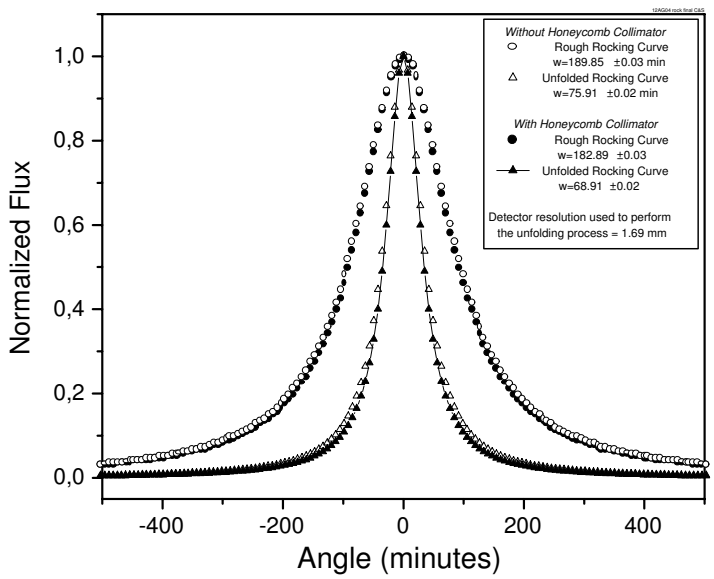

FIG. 6: Convoluted and unfolded RCs acquired with and without a honeycomb collimator. The beam divergence expressed by its RC has a FWHM of $1^{\circ} 16^{\prime}$.
[1] M. I. Silvani, Computed Tomography with thermal neutrons and a Position Sensitive Detector, DSc. Thesis, COPPE/UFRJBrazil(2001).In Portuguese.

[2] M. I. Silvani, R. T. Lopes, E. F. Jesus, G. L. de Almeida, and A. F. Barbosa, Nucl. Instr. Meth. in Phys. Research A 505, 568 (2003).

[3] M. I. Silvani, G. L. de Almeida, R. C. A. A. Furieri, R. T. Lopes, E. O. de Jesus, and A. F. Barbosa, Braz. J. Phys. 33, 286 (2003).

[4] M. I. Silvani, R. T. Lopes, E. F. Jesus, G. L. de Almeida, A. F. Barbosa, and D. Braz, Conversion of a X-ray Position Sensitive Detector for use in Thermal Neutron Tomographic Systems, V
International Topical Meeting on Industrial Radiation and Radioisotope Measurement Applications, Bologna, Italy (2002).

[5] M. J. Gonçalves, R. T. Lopes, M. I. Silvani, G. L. Almeida, and R. Furieri, Braz. J. Phys. 34, 756 (2004).

[6] G. L. de Almeida, M. I. Silvani, R. C. A. A. Furieri, R. T. Lopes, and M. J. Gonçalves, Braz. J. Phys. 34, 767 (2004).

[7] ASTM E 1441-95 and 1570-95a, Non-destructive Testing, Radiation Methods, computed Tomography, Guide for Imaging and Practice Examination, ISO/TC 135/SC5, N118 (1996). 